Navin Gupta, Emrecan Dilmen und Ryuji Morizane

\title{
3.6 3-D-Nierenorganoide für die Translation neuen Wissens vom Labor in die Klinik („,bench to bedside“)32
}

Zusammengefasst und aus dem Englischen übersetzt von Lilian Marx-Stölting

Nieren sind lebenswichtige Organe, die das Blut filtern und das dynamische Wasserund Elektrolyt-Gleichgewicht (Homöostase) des Körpers aufrechterhalten. Sie weisen dabei eine erstaunliche Plastizität und Flexibilität auf. Da die Niere von Säugetieren (und damit auch die des Menschen) ein nicht-regeneratives Organ ist, sich also nicht selbst erneuern kann, akkumuliert sich der Verlust ihrer funktionellen Einheiten, der Nephrone, im Verlauf der Zeit in allen Individuen. Weit verbreitete systemische Erkrankungen wie hoher Blutdruck und Diabetes mellitus, die Einnahme von Medikamenten oder akute Nierenschädigungen sowie primäre Krankheiten der Niere beschleunigen den Verlust der Nierenfunktion und verursachen chronische Nierenerkrankungen. In den USA kommen chronische Nierenerkrankungen mit einer Häufigkeit von $14 \%$ in der Bevölkerung vor und führen zu jährlichen Behandlungskosten von 120 Mrd. US\$. Die Lebensqualität der Erkrankten verringert sich erheblich und es kommt im fortgeschrittenen Stadium zu einer irreversiblen Niereninsuffizienz, die eine Nierenersatztherapie in Form regelmäßiger Dialysen oder einer Nierentransplantation erforderlich macht. Die Kosten für Dialysen machen in den USA $7 \%$ des Budgets der öffentlichen Krankenversicherung Medicare aus, Nierentransplantationen hingegen sind durch den Mangel an passenden Spenderorganen nur begrenzt durchführbar. Nierenkrankheiten stellen somit eine enorme Belastung für die Gesundheitssysteme und die betroffenen Patientinnen und Patienten dar.

In Anbetracht dieser Problemlage bestehen hohe Erwartungen an die seit Kurzem mögliche Herstellung von Nierenorganoiden auf Basis von humanen pluripotenten Stammzellen (hPS-Zellen). Nierenorganoide weisen eine hohe Ähnlichkeit zur

32 Dieser Beitrag ist eine deutsche Zusammenfassung des Review-Artikels „3D kidney organoids for bench-to-bedside translation" von Navin Gupta, Emrecan Dilmen und Ryuji Morizane, der 2020 im Journal of Molecular Medicine erschienen ist und eine umfangreiche Darstellung des wissenschaftlichen Sachstands sowie der aktuellen Literatur zum Thema (Stand Frühjahr 2020) bietet. 
menschlichen Niere auf und können für Toxizitätsstudien, für die personalisierte Medizin und für Medikamententests, für die regenerative Medizin oder als Modellsysteme für die Erforschung der Entwicklung und Erkrankung der Niere genutzt werden. Eine voll funktionsfähige Niere kann jedoch bislang noch nicht künstlich aus pluripotenten Stammzellen hergestellt werden, obwohl bereits vielversprechende Studien vorliegen. Es besteht die Hoffnung, dass transplantierbare Nierenorganoide eines Tages eine Alternative zu Dialyse und Nierentransplantationen darstellen könnten. Trotz dieses großen Potenzials von Nierenorganoiden bleiben einige Herausforderungen bestehen, wozu insbesondere ihre Unreife, begrenzte Reproduzierbarkeit sowie der Mangel eines integrierten funktionsfähigen Gefäßsystems gehören. Bei unreifem in vitro induziertem Gewebe liegen Zellen zum Teil noch undifferenziert vor, sodass das Gewebe nicht alle für die Funktion notwendigen Zelltypen in funktionsfähigem Zustand enthält. Um funktionstüchtig zu sein, müssen die Teilsysteme (Durchblutung, Blutgefäße, abführende Leitsysteme etc.) miteinander erfolgreich interagieren. Sie müssen also alle in einem Organoid integriert sein und zusammenarbeiten. Es wird derzeit noch nach Wegen gesucht, den Reifungsprozess von Organoiden zu verbessern (siehe auch Frum/ Spence, Kap. 3.1). Auch die Reproduzierbarkeit von Organoiden ist eingeschränkt, da viele Entwicklungsschritte nacheinander durch Zugabe verschiedener Faktoren durchlaufen werden und es dabei zu individuellen Unterschieden zwischen den erzeugten Organoiden kommen kann.

Im Folgenden wird beschrieben, wie neuere Erkenntnisse in der Entwicklungsbiologie der Niere die Forschung an Nierenorganoiden beeinflusst haben. Danach wird über Möglichkeiten berichtet, wie aktuell auftretende Schwierigkeiten und Hürden bezüglich der klinischen Umsetzung überwunden werden könnten.

Herkömmliche Forschungsmodelle wie zweidimensionale Zellkulturen und Tiermodelle erfassen die komplexe Funktion der menschlichen Niere in vivo nur unzureichend, ihre Ergebnisse sind daher nur begrenzt für die klinische Anwendung nutzbar. Aus diesem Grund sind Nierenorganoide für die Forschung von besonderer Bedeutung. Sie lassen sich aus hPS-Zellen, d. h. aus humanen embryonalen Stammzellen (hES-Zellen) oder aus humanen induzierten pluripotenten Stammzellen (hiPS-Zellen) in vitro generieren (siehe auch Einleitung, Kap. 2.1). Letztere sind besonders für die personalisierte Medizin geeignet, da sie patientenspezifisch, also aus Zellen zu behandelnder Patientinnen und Patienten, generiert werden können.

Will man ein Nierenorganoid in vitro herstellen, das tatsächlich wie eine Niere funktioniert, benötigt man mehr als ein Dutzend spezifischer, voneinander unterscheidbarer Zelltypen, aus denen ein Nephron in vivo besteht. Zur Herstellung eines voll funktionsfähigen Nephrons müssten alle dazu beitragenden Zelltypen aus einem 
einzigen Vorläufer generiert werden. Dies kann nur über eine angeleitete schrittweise Differenzierung erfolgen, die umfangreiches Wissen über die natürliche Nierenentwicklung voraussetzt. Der nötige Ansatz müsste die gerichtete Differenzierung einer Niere im Körper nachahmen und sich an den natürlich im Körper ablaufenden Prozessen orientieren.

\subsubsection{Nierenentwicklung}

Die natürliche Nierenentwicklung ist der Goldstandard für die gerichtete Differenzierung, mit der in vitro Gewebe erzeugt wird, das seinen In-vivo-Gegenstücken möglichst ähnlich ist. Jahrelange Forschung hat gezeigt, dass bestimmte Signalwege die Entwicklung, Gewebestruktur und Induktion von Organvorläufern während der Embryonalentwicklung steuern (siehe auch Frum/Spence, Kap. 3.1). Diese Signalwege sind wesentlich für die kontinuierliche Entwicklung von Geweben aller drei Keimblätter (Entoderm, Mesoderm und Ektoderm, die Niere wird aus dem Mesoderm gebildet; siehe auch Einleitung, Kap. 2.1) während der Embryonalentwicklung. In der Säugerniere durchläuft die Entwicklung der Nephrone drei Stadien: Pronephros, Mesonephros und Metanephros. Metanephros bezeichnet die letzte Entwicklungsstufe der Nierenentwicklung - die ausgewachsene Niere. Für die Induktion nierenspezifischer Zelllinien in gerichteten Differenzierungsprotokollen sind die Reihenfolge und biochemischen Eigenschaften der Signalwege sowie die zeitliche Aufeinanderfolge von Zwischenstadien von wesentlicher Bedeutung.

\subsubsection{3-D-Nierenorganoide}

Diese entwicklungsbiologischen Erkenntnisse in Verbindung mit fortgeschrittenen Techniken der gerichteten Differenzierung von hPS-Zellen ermöglichten die Herstellung dreidimensionaler Nierenorganoide, also von Mini-Nieren in einer In-vitro-3-DMikroumwelt. Dabei wird mittels Transkriptionsfaktoren und Zusätzen im Nährmedium nacheinander die Bildung verschiedener Vorläuferzellen angeregt. Diese ähneln menschlichen Nieren in Gewebeaufbau, Zellzusammensetzung und Differenzierung. Mit den neuen Methoden der Einzelzellanalytik ${ }^{33}$ lässt sich zudem die Entwicklung der einzelnen Stammzellen bis hin zum Organoid nachverfolgen. Zellen so generierter

33 Siehe hierzu Walter/Schickl (2019): Einzelzellanalyse in Forschung und Medizin. Eine Stellungnahme der interdisziplinären Arbeitsgruppe Gentechnologiebericht. Kostenlos abrufbar unter: https:/ /www.gentechnologiebericht.de/fileadmin/user_upload/Webseitendateien/Dokumente/BBAW_Einz elzellanalyse_A5_PDF-A1-b.pdf [19.05.2020]. 
Organoide weisen ähnliche Genexpressionsmuster auf wie Zellen früher embryonaler Nieren. Die Erkenntnisse der Nierenentwicklung beeinflussen jedoch nicht nur die Herstellung von Organoiden, die Forschung an Organoiden trägt ihrerseits dazu bei, diese Entwicklungsprozesse besser zu verstehen. So können beispielsweise Hypothesen über die notwendigen Faktoren für eine normale Nierenentwicklung gebildet und an Organoiden getestet werden.

\subsubsection{Herausforderungen und Ausblick}

Eine große Herausforderung für die Erforschung der Nierenentwicklung besteht darin, das Zusammenspiel von Blutgefäßen mit anderen Zellen und abführenden Leitungssystemen besser $\mathrm{zu}$ verstehen und in Organoiden nachzubilden. Die Bildung von Blutgefäßen in den Nieren ist in zwei voneinander getrennte Prozesse untergliedert: die Vaskularisierung (Neubildung von Blutgefäßen) und die Angiogenese (Blutgefäßentwicklung aus bereits angelegten Blutgefäßen, etwa in der Embryogenese, der Wundheilung oder bei Krankheiten). Die genauen Wechselwirkungen und das Zusammenspiel beider Prozesse sind noch unbekannt. Bisherige Organoide enthalten grundsätzlich keine funktionsfähigen Blutgefäße. Erste 3-D-Nierenorganoide enthielten allerdings wenige vaskuläre Zellen, die die Bildung kleiner Blutgefäße nachahmten, wie sie in sich entwickelndem Nierengewebe stattfindet. Die Glomeruli (filternde Bereiche der Nephrone) blieben dabei aber ohne Blutgefäße. Dagegen konnten an transplantierten Organoiden im Tierversuch Blutgefäße in den Glomeruli nachgewiesen werden. Derartige Versuche zur Bildung von Blutgefäßen sind vielversprechend und laufen derzeit noch. Die mangelnde Filtrationsleistung der Nierenorganoide aufgrund fehlender Durchblutung sowie ein Mangel an abführenden Leitungssystemen für das Filtrat ist ein Problem von Nierenorganoiden, das bislang noch nicht gelöst werden konnte. Trotz der großen Erfolge der letzten Jahre werden verbesserte Protokolle und Modifikationen benötigt, um Probleme der Gewebereifung, eines mit Blut durchströmbaren Gefäßsystems und der Systemintegration sowie der Reproduzierbarkeit lösen zu können

In Verbindung mit Organ-on-a-chip-Technologien (siehe auch Einleitung, Kap. 2.1) könnten Nierenorganoide auch zur klinischen Nutzung gelangen (siehe Abbildung 1). Durch unser zunehmendes Wissen über die Nierenentwicklung und die Kombination mit diesen neuen Techniken sind in jedem Fall große Fortschritte in der Nierenorganoidforschung zu erwarten. 
Abbildung 1: Zur Anwendung von Nierenorganoiden

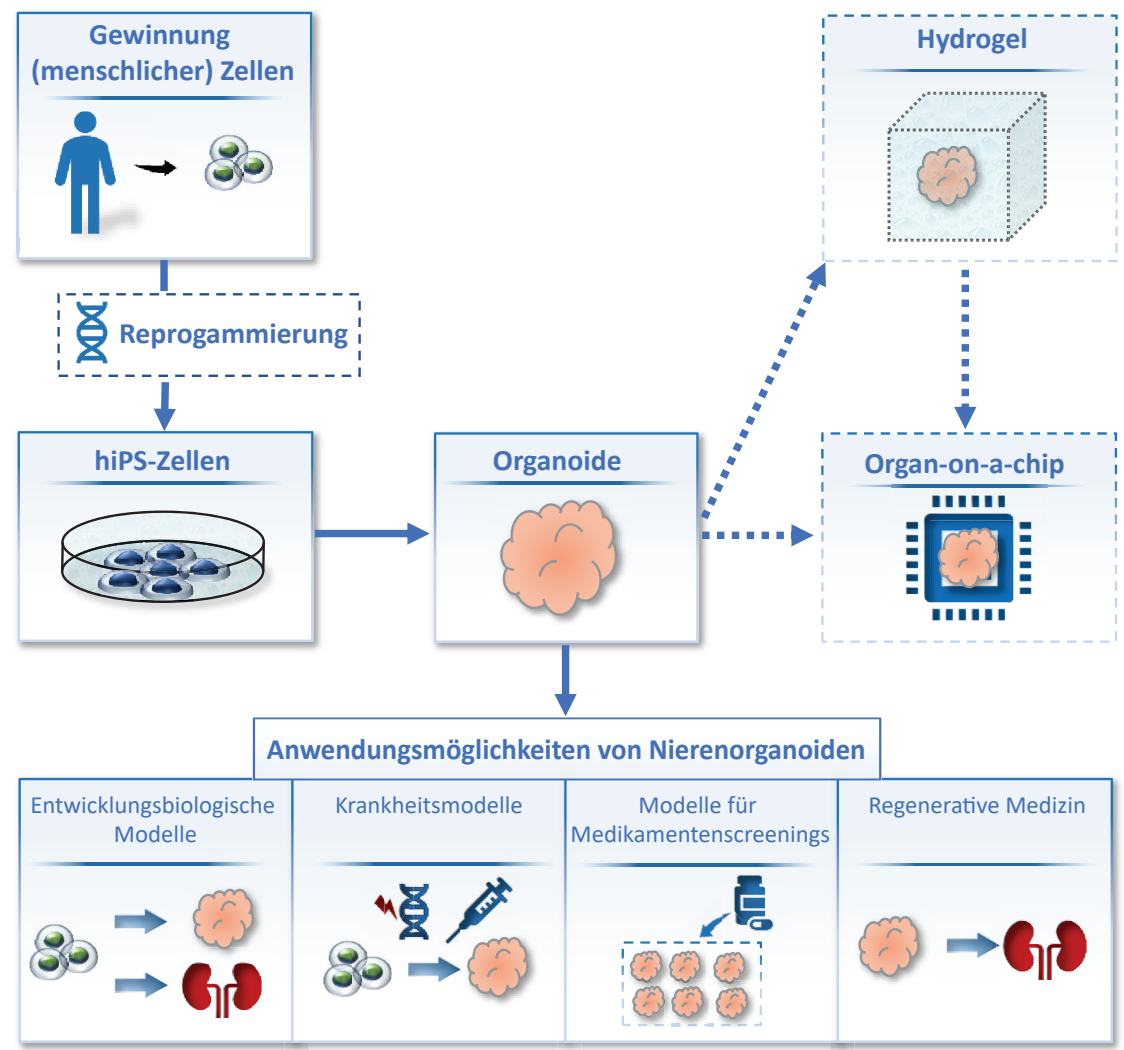

Humane induzierte pluripotente Stammzellen (hiPS-Zellen) können von Patientinnen und Patienten gewonnen werden, um (patientenspezifische) Nierenorganoide herzustellen. Dabei können auch genetische Modifikationen eingeführt werden, um die Organoide als Krankheitsmodelle oder zur Erprobung genetischer Therapien einsetzen zu können. Die erhaltenen Nierenorganoide können in Hydrogelen und/oder Organ-on-a-Chip-Systemen verwendet werden, um eine größere Ähnlichkeit mit der Situation in vivo herzustellen. Als Entwicklungsmodelle können sie dazu genutzt werden, um neue Erkenntnisse zur Nierenentwicklung zu generieren und Nierenmodelle zu verbessern. Organoide Krankheitsmodelle können entweder durch genetische Veränderung oder durch Kontakt mit krankheitsauslösenden Stoffen hergestellt und zur Entwicklung neuer Therapien eingesetzt werden. Nierenorganoide bieten auch eine wertvolle Plattform zur Durchführung von Medikamentenscreenings und Toxizitätstests in großem Maßstab. Schließlich können sie auch zum Ziel der Wiederherstellung einer funktionierenden Niere in der regenerativen Medizin beitragen. 